\author{
Mario Meglio \\ Beatrice Cioni \\ Gianpiero Tamburrini \\ Luigi Pentimalli \\ Yi Ma
}

\section{Surgical treatment of trigeminal neuralgia: results of percutaneous treatment versus microsurgical decompression}

Received: 22 August 2000

Accepted in revised form: 22 January 2001

M. Meglio (凶) • B. Cioni

G. Tamburrini • L. Pentimalli • Y. Ma

Institute of Neurosurgery,

Catholic University Medical School,

Largo A. Gemelli, 8, I-00168 Rome, Italy

e-mail: iclnc@rm.unicatt.it

Tel.: +39-06-30155469

Fax: +39-06-3051343

\begin{abstract}
A patient with trigeminal neuralgia may need different forms of treatment during his or her lifetime. Physicians should be aware of the different available surgical treatments, and know their effectiveness, side effects and complications.

Microvascular decompression is considered by many to be the most effective treatment. The goal of the procedure is to remove the cause of pain, obtained by decompressing the nerve at its entry point into the pons. Percutaneous procedures are more easily performed but the recurrence rate of pain is higher. It is difficult to compare the results of surgical procedures reported by different authors. Therefore, we compare the efficacy
\end{abstract}

\begin{abstract}
of 155 microvascular decompressions with 113 radiofrequency thermocoagulations and 215 percutaneous microcompressions performed by the same surgical team. Our study confirms microvascular decompression as the most effective surgical treatment, although percutaneous procedures play an important role in the treatment protocol and have to be offered to patients as a therapeutic option.
\end{abstract}

Key words Trigeminal neuralgia • Microvascular decompression • Percutaneous treatment

\section{Introduction}

Trigeminal neuralgia (TN), also known as "tic doloureux", is a well known pain condition, characterized by repetitious paroxysms of unilateral facial pain. The estimated incidence is four cases per 100000 persons [1]. Typically the pain is sudden in onset, severe in degree and short in duration. It is remittent, and with time, tends to become more and more frequent. Pain appears localized in one or more divisions of the fifth cranial nerve; more commonly it affects the lower part of the face. Spontaneous in onset, pain can also frequently be triggered by non-painful stimuli, such as touching, chewing, or talking.

The mean age at onset of TN is around 50 years, but it is becoming not so unusual to see cases of TN in younger patients. Carbamazepine is the most effective drug used in the treatment of TN and many patients are satisfactorily treated for their lifetime with pharmacological treatment. Unfortunately, with time, nearly one-half of patients either become unresponsive to medical treatment or complain of intolerable side effects, thus requiring a surgical procedure.

Among the surgical options routinely available for the treatment of TN, microvascular decompression (MVD) is the only one aimed at removing the putative cause of the disease (a conflicting vessel at the root entry zone in the pons) [2]. Such procedure, although requiring an open surgical approach, constitutes the only available conservative treatment, as it does not imply any damage to the nerve. On the contrary, other procedures, such as peripheral neurectomies and gasserian ganglion lesions performed with heat (radiofrequency), with chemicals (glycerol) or mechanically 
(balloons), ignore pain etiology and are aimed at producing a lesional impairment of nerve function. The main advantages of such destructive procedures are basically two: they can be performed with percutaneous techniques, and they have an acute success rate comparable to that achieved by MVD [3-13]. It is more difficult to compare the outcome of the different procedures concerning long-term success rates, complications and side effects, especially when performed by different surgical teams [3-13]. Moreover, the majority of papers report on the results of a single procedure (Table 1).

This report presents our experience with MVD and with two percutaneous procedures: percutaneous microcompression (PMC) and radiofrequency thermocoagulation of the gasserian ganglion (TCG). Reliable data in different procedures obtained by the same surgical team will help to choose the surgical strategy. Our algorithm for the treatment of trigeminal neuralgia is discussed.

\section{Patients and methods}

We surgically treated 483 consecutive patients for typical trigeminal neuralgia at Catholic University in Rome, between January 1982 and December 1998. There were 271 men and 212 women. The majority of patients were between 50 and 80 years, but 18 patients were between 30 and 40 years of age (Fig. 1). In 266 cases, trigeminal neuralgia affected the right side. In 151 cases pain was confined to the second and third divisions, in 118 cases it involved all trigeminal branches, and in 81 cases pain was limited to the third branch. No sensory or motor trigeminal deficit was evident at clinical examination. All patients underwent a neuroradiological study (CT and/or MRI) to rule out the presence of tumors or demyelinating lesions as the cause of trigeminal neuralgia.

MVD was recommended for patients in good general condition. Percutaneous procedures were recommended for patients in poor general condition or in patients who did not accept open surgery. PMC was usually prefered as first treatment because of its simplicity. TCG was considered in patients with isolated or in predominant division III neuralgia and was contraindicated in cases of isolated or predominant division I pain. Overall, 155 patients were submitted to MVD of the trigeminal nerve, 113 to TCG and 215 to PMC of the gasserian ganglion.

MVD was performed through a small lateral posterior fossa craniectomy (retromastoid craniectomy) placed just below the transverse sinus. Patients were in the supine position with the head flexed and rotated towards the opposite site. The nerve was approached with microsurgical technique by a supracerebellar infratentorial route. Cerebellar retraction was minimal under these conditions and after cutting the petrosal vein, it was possible to explore completely the fifth cranial nerve, from its merge at the pons to its passage in the middle cranial fossa. Any compressing artery and eventually some veins were mobilized and repositioned with teflon felts; other compressive veins were coagulated and divided. In 28 cases no clear neurovascular conflict was evident at the operation; in 12 of them trigeminal neurolysis was performed. MVD was performed as first surgical procedure in 84 cases, while 71 patients had previously undergone one or more ablative procedures (e.g. radiofrequency rhizotomy, balloon compression, glycerol rhizotomy).

TCG was performed according to the technique described by Sweet and Wepsic [14]: a percutaneous approach was used in order to reach the foramen ovale and to position the electrode's tip under the assistance of electrical sensory and motor stimulation. The lesion was obtained by means of a temperature of $75-80{ }^{\circ} \mathrm{C}$ held

Table 1 Acute and long-term success rates for procedures used to treat trigeminal neuralgia. Values refer to studies involving 51-1185 patients. No significant differences among success rates for the four procedures were noted

\begin{tabular}{llcr}
\hline Procedure & Acute success rate, $\%$ & Long-term success rate, $\%$ & Reference \\
\hline MVD & 80 & 71 & {$[7]$} \\
& 98 & $70]$ & {$[13]$} \\
& 96.7 & 82.5 & {$[3]$} \\
PMC & 79 & 45 & {$[8]$} \\
& 94.6 & 69.5 & {$[5]$} \\
TCG & 78 & 63 & {$[11]$} \\
& 89 & 78.6 & {$[17]$} \\
Glycerol injection & 83 & 49 & {$[4]$} \\
& 93 & 36.7 & {$[6]$} \\
\hline
\end{tabular}

$M V D$, microvascular decompression; $P M C$, percutaneous microcompression; $T C G$, thermocoagulation 
Fig. 1 Age distribution of the 483 trigeminal neuralgia patients surgically treated at Catholic University in Rome, between January 1982 and December 1998

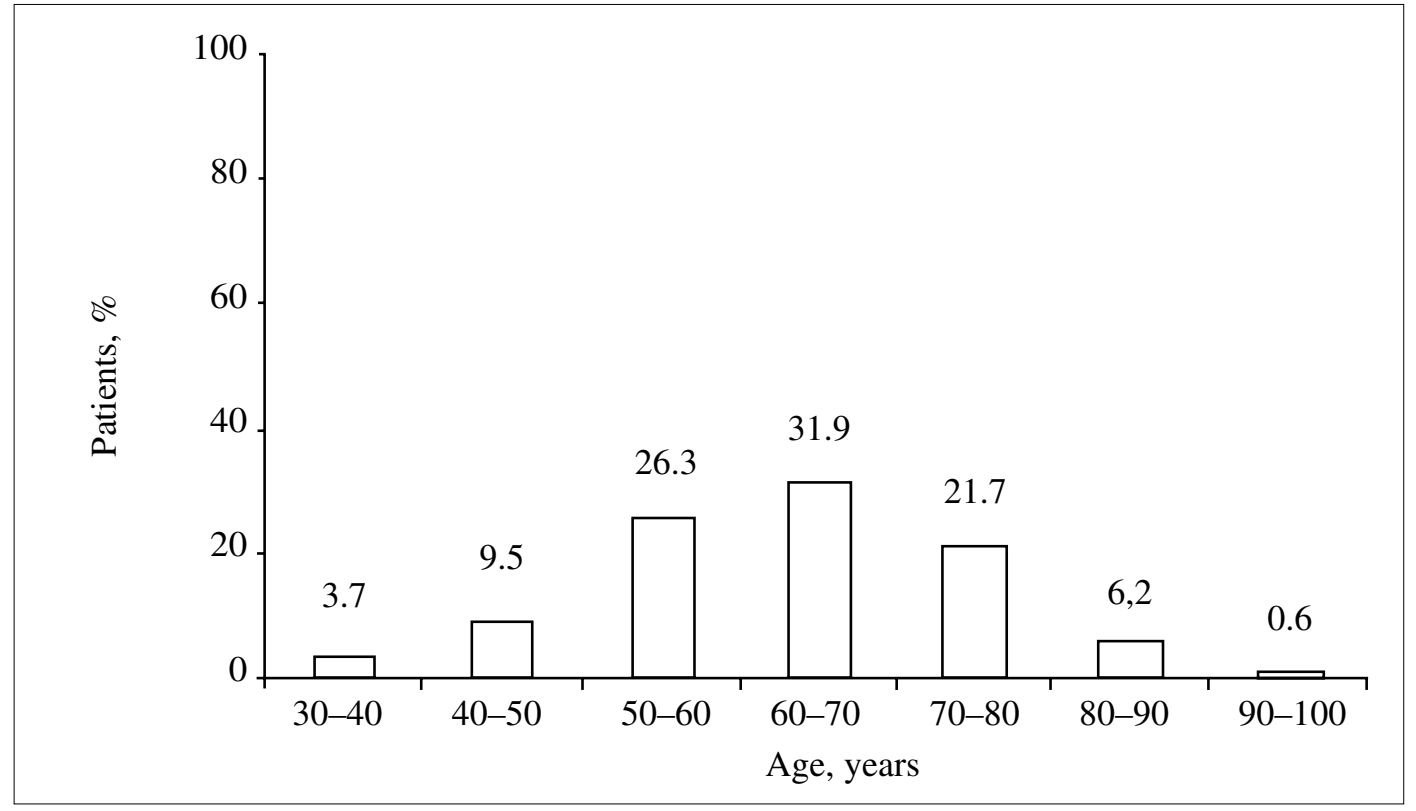

for 1-3 minutes. Radiofrequency rhizotomy was the first surgical procedure in 43 patients, while 70 patients previously underwent other trigeminal procedures: percutaneous ablative surgery in 50 cases, MVD in 7 cases, and both ablative procedures and MVD in 13 cases.

PMC was performed according to the technique described by Mullan and Lichtor [15]: under fluoroscopy, a \#4 Fogarty arterial catheter was introduced into the Meckel cave, through a bluntedged thin-walled cannula. The balloon was then inflated with 0.75 $\mathrm{ml}$ contrast material for 1-10 minutes. This was the first surgical procedure in 103 patients, while it was adopted as second treatment in 112 cases: percutaneous ablative surgery in 95 cases, MVD in 7 cases, and both ablative procedures and MVD in 10 cases.

The analyzed outcome parameters included technical success (completion of the procedure), pain relief and its recurrence, and complications. Pain relief was evaluated, as reported by patients, on a visual analogue scale, and graded as: complete pain relief, no pain, no medications; partial pain relief, at least $50 \%$ analgesia (VAS $\leq 5$ ), medication needed; poor pain relief, less than $50 \%$ analgesia (VAS > 5), other surgical maneuvers needed. Immediate pain relief was evaluated within 1 week of the operation. Long-term results were assessed by clinical follow-up and telephone surveys performed by physicians not involved in the study. Mean follow-up was 45.4 months, ranging from 1 month to 168 months. The occurrence of poor pain relief was considered a failure and ended the follow-up. Furthermore, for each considered procedure, we divided patients into different groups, depending on whether the procedure was adopted as a first- or second-choice treatment. Statistical analysis was carried out to compare the outcome parameters in the 3 different procedures utilizing the $\chi^{2}$ test.

\section{Results}

Microvascular decompression

MVD was performed on 155 patients aged 30-82 years (mean, 60.3 years). In 127 patients, a neurovascular conflict was identified. It originated from an artery in 111 cases, from a vein in 5 cases and from an artery plus a vein in 11 cases. Appropriate decompression was achieved in 123 cases. In the remaining 4 cases the compression was from a megadolichobasilar artery and it was not possible to securely and stably distance it from the nerve. In 28 patients no clear compression was evident; in 12 of these cases, trigeminal neurolysis was performed.

At hospital discharge (Fig. 2), MVD had accomplished complete pain relief in 118 patients $(76.1 \%)$, partial relief in $30(19.3 \%)$ and poor relief in $7(4.5 \%)$. There were 99 patients available for follow-up (mean, 52.5 months). Of these, $58(58.5 \%)$ were completely pain free, $27(27.3 \%)$ had partial pain control, and in 14 cases $(14.1 \%)$ the procedure had failed (Fig. 3). The mean recurrence time was 12.7 months.

The 4 patients in whom an appropriate decompression was technically impossible reported complete pain relief at discharge. At follow-up (mean, 33 months), 2 of the 3 available patients still reported complete pain relief $(66.6 \%)$. This success rate was not significantly different from that achieved in the whole MVD patient population. 
Fig. 2 Pain relief at hospital discharge after surgical treatment for trigeminal neuralgia. A total of 155 patients received microvascular decompression $(M D V), 215$ patients were trested with percutaneous microcompression $(P M C)$ and 113 patients had radiofrequency thermocoagulation (TCG). No significant difference was evident comparing the results of the three procedures

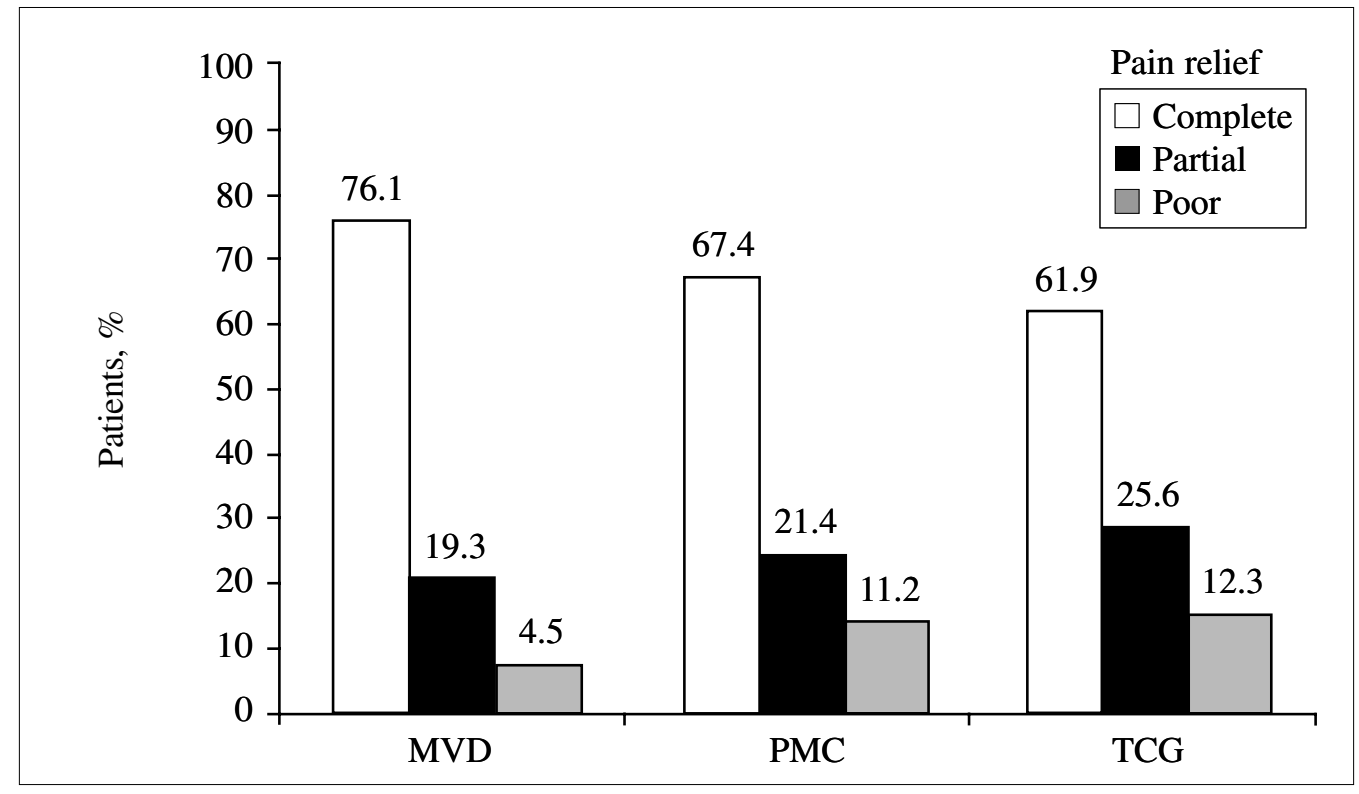

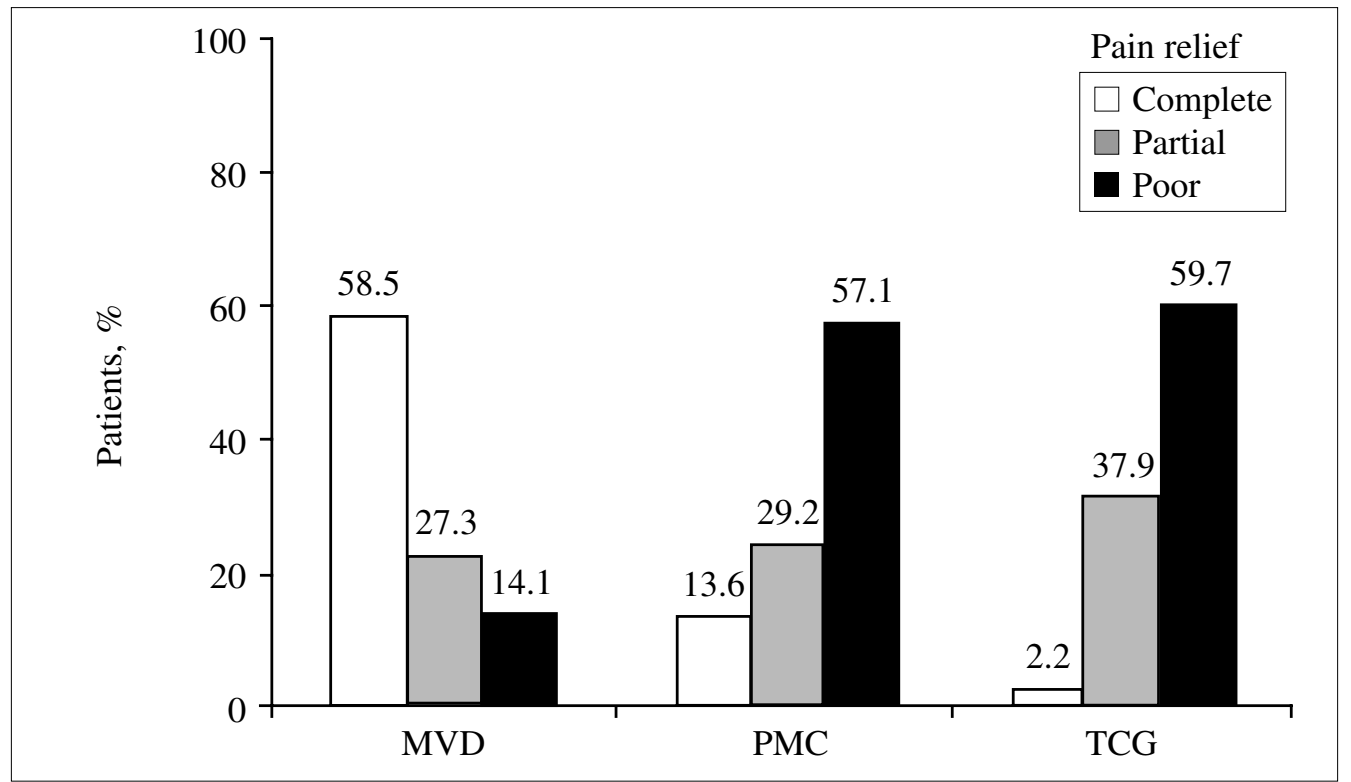

Fig. 3 Pain relief at followup after MVD, PMC and TCG. The percentage of patients reporting complete pain relief after MVD was significantly higher than after PMC or TCG
The acute and long-term outcomes in patients in whom no clear compression was evident at surgery were not significantly different from that obtained in the whole MVD population. The trigeminal neurolysis did not significantly influence the results.

MVD was performed as first procedure in 84 patients and produced complete pain relief in $65(77.3 \%)$, partial relief in 15 $(17.8 \%)$ and poor pain relief in $4(4.8 \%)$. There were 54 patients available for follow-up (mean follow-up, 45.9 months). Of these, $32(59.2 \%)$ had complete pain relief, $16(29.6 \%)$ referred a partial relief and $6(11.1 \%)$ had poor pain relief.
MVD was performed after one or more ablative procedures in 71 cases. At hospital discharge 53 patients (74.6\%) had complete pain relief, $15(21.1 \%)$ referred a partial relief and $3(4.2 \%)$ had poor pain relief. There were 45 patients available for follow-up (mean follow-up, 54.7 months). Complete pain relief was observed in 26 cases $(57.7 \%)$, partial relief in 11 patients $(24.4 \%)$ and poor pain relief in 8 patients $(17.9 \%)$. The acute and long-term results achieved by MVD as first surgical therapy were not statistically different from those achieved by MVD after one or more ablative procedures. 
Among the patients receiving MVD, 34 (21.9\%) reported complications requiring surgical or medical treatment: 2 supratentorial hematomas $(1.2 \%), 4$ wound infections $(2.5 \%), 2$ cases of meningitis $(1.2 \%)$, and 25 cerebrospinal fluid (CSF) fistulae (16.1\%). In one $(0.6 \%)$ of our first cases, cerebellar infarction led to death. Permanent morbidity was represented by 2 cases of hearing dysfunction $(1.2 \%)$ and by 3 cases of trigeminal hypoesthesia (1.9\%).

\section{Thermocoagulation}

TCG was performed in 113 patients aged 36-87 years (mean age, 59.8 years). In 95 patients $(84.1 \%)$, the procedure generated trigeminal hypoesthesia. At discharge (Fig. 2), complete pain relief was reported by 70 patients $(61.9 \%)$, partial relief by $29(25.6 \%)$ and poor relief by $14(12.3 \%)$.

There were 87 patients available for follow-up (mean, 45.4 months). Only two patients $(2.2 \%)$ were completely pain-free at follow-up (Fig. 3). Partial pain relief was reported in 33 cases $(37.9 \%)$ while poor relief was referred by 52 patients $(59.7 \%)$. Mean recurrence time was 21.5 months.

The 18 patients in whom postoperative hypoesthesia was not observed did significantly worse $(p<0.001)$ than the whole TCG population, at both short- and long-term follow-up.

TCG was performed in 43 patients as first treatment and achieved complete relief in $29(67.4 \%)$, partial relief in 9 $(20.9 \%)$, and poor relief in $5(11.6 \%)$ cases. Of these, 35 patients were available for follow-up (mean follow-up, 47.3 months): 14 patients (40\%) referred a partial pain relief and $19(54.2 \%)$ a poor relief; 2 patients $(5.7 \%)$ still referred a complete pain relief.

TCG was performed after one or more previous procedures in 70 cases. Complete pain relief was obtained in 41 patients $(58.6 \%)$, partial relief in 20 cases (28.6\%) and poor relief in 9 cases $(12.8 \%)$. There were 52 patients available for clinical follow-up (mean, 37.3 months); 19 of these patients $(36.5 \%)$ referred a partial pain relief and $33(63.5 \%)$ a poor relief. The results after TCG as first surgical treatment were not statistically different from those following TCG after previous procedures.

Among the patients receiving TCG, 20 (17.6\%) reported marked dysesthesia requiring medical and/or surgical treatment. Disturbing masticatory muscles weakness was reported by 1 patient $(0.8 \%)$.

\section{Percutaneous microcompression}

PMC was performed in 215 patients aged 33-94 years (mean, 65.9 years). In 7 of them the procedure was not completed because of repeated balloon explosion (4 cases) or because the technical impossibility to enter the foramen ovale with the needle (3 cases).

At hospital discharge (Fig. 2), complete pain relief was achieved in 145 patients $(67.4 \%)$, partial pain relief in 46 $(21.4 \%)$ and poor pain relief in $24(11.2 \%)$.

There were 154 patients available for follow up (mean, 38.3 months). Of these $21(13.6 \%)$ were completely pain free, $45(29.2 \%)$ had a partial control of pain and $88(57.1 \%)$ referred a poor pain relief (Fig. 3). Mean recurrence time was 17.1 months.

PMC was the first treatment for 103 patients who reported complete pain relief in 79 cases $(76.7 \%)$, partial relief in $16(15.5 \%)$ and poor relief in 8 patients $(7.7 \%)$. Of these 103 patients, 74 were available follow-up study (mean, 39.6 months): $15(20.2 \%)$ referred a complete pain relief, 24 $(32.4 \%)$ a partial relief and $35(47.3 \%)$ a poor relief.

PMC was performed after one or more previous procedures in 112 patients. Complete pain relief was observed in 66 cases $(58.9 \%)$, partial relief in 30 patients $(26.8 \%)$, and poor relief in 16 cases $(14.3 \%)$. There were 80 patients available for follow-up (mean, 32.8 months): complete pain relief was referred by 6 patients $(7.5 \%)$, partial by 21 $(26.2 \%)$ and poor by 53 patients $(66.2 \%)$. No significant difference was noted comparing the results obtained by PMC as first treatment or after one or more previous procedures.

A total of 11 patients $(5.1 \%)$ complained of marked dysesthesia requiring subsequent treatment; 2 patients $(0.9 \%)$ were disturbed by a marked weakness of masticatory muscles.

We compared the complete pain relief rate in the three different procedures. At hospital discharge, no statistical difference was evident among MVD, TCG and PMC rates of complete pain relief. In the long term, MVD patients did significantly better $(p<0.001)$ than PMC and TCG patients. We analyzed the results obtained by the three different procedures in patients with neuralgia confined to a single division, in order to compare the efficacy of the different procedures on the different branches. At hospital discharge, no significant difference was evident between the three procedures, whereas, at long term, MVD was more effective than the percutaneous procedures, regardless of the involved division.

\section{Discussion}

Percutaneous destructive procedures and MVD are rarely handled by the same author and the reports comparing the results of the different treatments are based on literature data. The point is that these data are not comparable; in fact, what is considered the goal of the procedure for one author may be considered to be an unwanted side effect for another. Taha and Tew [11] compared the results of 500 radiofrequency rhizotomies performed at the University of Cincinnati with the results of large series reported in the lit- 
erature including 6205 radiofrequency rhizotomies, 1217 glycerol rhizotomies, 759 balloon compressions, 1417 MVD and 250 partial trigeminal rhizotomies. They concluded that radiofrequency rhizotomy is the procedure of choice for most patients undergoing first surgical treatment, unless the patient is a healthy subject with isolated pain in the first division and/or he desires no sensory deficit; in these cases MVD is recommended. The results reported in their 500 radiofrequency rhizotomies were the following: initial pain relief $98 \%$, pain recurrence (same distribution of the initial trigeminal pain or in an adjacent trigeminal division) $20 \%$, facial numbness $98 \%$, minor dysesthesia $9 \%$, major dysesthesia $2 \%$, anesthesia dolorosa $0.2 \%$, corneal anesthesia $3 \%$, keratitis $0.6 \%$, trigeminal motor dysfunction $7 \%$, perioperative morbidity $0.6 \%$, perioperative mortality $0 \%$. Importantly, in their procedure the goal was "dense hypoalgesia defined as loss of two-thirds or more of pinprick perception". In a clinical study published in Neurosurgery in 1997, Kondo [13] reported the results of MVD in 281 patients divided into two series, the first one of $127 \mathrm{TN}$ patients with a mean follow-up of $12.6 \pm 2.1$ years and the second one of 154 patients with a mean follow-up of $7.0 \pm 1.4$ years. Immediate postoperative cure rates were respectively $92.9 \%$ and $96.7 \%$, becoming at the follow-up $80.3 \%$ and $82.5 \%$ with recurrence rates of $10.2 \%$ and $6.5 \%$. The most significant complication was hearing dysfunction: $7.1 \%$ and $4.5 \%$ in the two series. An important parameter of success taken into account by this author was the patient's satisfaction rate, in fact "if some sensory impairment is present after MVD in patients with TN despite the complete cessation of pain, patients will not be completely satisfied with the results of surgery". So, trigeminal hypoesthesia is the goal of TCG, but is a cause of unsatisfaction for MVD, influencing in opposite ways the results.

The effort of this study has been to compare the outcome of three different procedures performed by the same surgical team and evaluated with the same criteria. Three parameters were considered to comparatively evaluate the three surgical procedures: (1) technical success; (2) outcome and pain recurrence; (3) side effects and postoperative complications. 1. A review of the more recent literature shows that thermocoagulation of the gasserian ganglion is actually performed with a technical success of $98 \%-100 \%[3,11,16$, 17]. Among the most relevant improvements of such methodology, since its first description by Sweet and Wepsic [14], are: the utilization of curved electrodes, so to obtain a better topographical localization of the pain site, and the use of perioperative CT in those patients in which a fluoroscopic identification of the foramen ovale appears difficult $[3,11,17]$. Procedures of microcompression of the gasserian ganglion are also related to a high percentage of technical success, ranging from $89 \%$ to $100 \%$ of cases $[3,8,11,16]$. In our series, microcom- pression of the gasserian ganglion was technically possible in $96.7 \%$ of cases, while TCG was completed in $84.1 \%$ of cases.

Analyzing recent literature (1667 cases), Taha and Tew [11] reported 85\% technical success after MVD procedures; similar rates were reported by Burchiel et al. [18] (82\%) and Walchenbach et al. [7] (86\%). Generally, the technical success through an MVD procedure is inferior to that through percutaneous procedures. Such data appear basically related to two main factors: a slight or absent neurovascular conflict and an evident, although not surgically correctable neurovascular conflict, i.e. from a megadolichobasilar artery $[11,19-21]$.

In our experience, technical success for MVD procedures was $79.4 \%$; in fact, $18.1 \%$ of patients presented at surgery no clear vascular conflict and $2.5 \%$ had a neurovascular conflict which was not surgically correctable.

2. Literature data (Table 1) show equal acute success rates after both percutaneous and MVD procedures (total or subtotal remission of pain: TCG, 81.8\%-95\%; PMC, 79\%-95.6\%; MVD, 80\%-100\%) [5, 8, 10, 11, 13, 17, 18, 22-24]. A long-term analysis appears more difficult to perform due to differences in describing results and to variability of considered follow-up. As a consequence, the long-term success rates reported by different authors are also similar for the different procedures.

Taha and Tew [11], after comparing their series of radiofrequency rhizotomies with the results of large series of percutaneous procedures (radiofrequency rhizotomies, balloon compressions and glycerol rhizotomies) and with MVD, reported a success rate of $79 \%$ after PMC (mean follow-up, 4 years), $80 \%$ following TCG procedures (mean follow-up, 9 years), and $85 \%$ after MVD (mean follow-up, 5 years). Therefore the authors did not find significant differences in the outcomes among the three therapeutic options. Our experience confirms a basic similarity in the acute results, when comparing percutaneous procedures with MVD. The rates of complete pain remission were respectively PMC 67.4\%, TCG 61.9\%, and MVD 76.1\%. However, at the long-term follow-up (mean, PMC 38.3 months; TCG 45.4 months and MVD 52.5 months), a significant difference was evident among the outcomes after the three procedures. While long-term follow-up revealed still acceptable results after MVD (complete pain relief in $58.5 \%$ of cases), a higher percentage of pain recurrence was noted after PMC (complete pain relief in $13.6 \%$ ) and after TCG (complete pain relief in $2.2 \%$ ). Moreover, our analysis was extended to compare the results after the three procedures performed as first or as second treatment. Regarding percutaneous procedures, we observed a decrease in the success rate, both acutely 
(complete pain relief, PMC $76.7 \%$ vs. 58.9\%; TGC $67.4 \%$ vs. $58.6 \%$ ) and at long-term follow-up (complete pain relief, $\mathrm{PMC}=20.2 \%$ vs. $7.5 \%$; TCG $5.7 \%$ vs. $0 \%$ ) that did not reach statistical significance. No significant success rate variations were noted when analyzing outcomes after MVD, both acutely (complete pain remission in $77.3 \%$ vs. $74.6 \%$ ) and at long-term follow-up (complete pain remission in $59.2 \%$ vs. $57.7 \%$ ). Such results are in contrast to the experience reported by others [7, 21, 25]. Recently, Barker et al. [10] described a worse prognostic role played exclusively by previous MVD procedures but not by previous percutaneous procedures. These authors indicated a worse long-term prognosis correlated to other factors, such as the lack of an immediate postoperative disappearance of pain, the presence of an unclear neurovascular conflict and preoperative signs lasting more than eight years. This last point was criticized by Klun [26] in whose experience no data matching symptoms duration with the postoperative success were evident. More general agreement is evident concerning the analysis of correlation between an unclear neurovascular conflict and pain persistence or recurrence $[10,26,27]$. In our study no outcome differences were observed between cases harboring a clear or an unclear neurovascular conflict. In 28 patients of our series no clear conflict was noted intraoperatively and 12 patients were submitted to neurolysis of the fifth nerve root while 16 underwent only posterior fossa exploration. No prognostic differences were noted at followup in the two groups. The evidence of a statistically similar outcome for patients with a clear and successfully treated conflict and for patients with an unclear or untreatable conflict, regardless of neurolysis, could induce, in agreement with Adams [28], that "The evidence used to support the hypothesis of microvascular conflict... is believed to be insufficient and unconvincing. The basis of MVD could be trauma of the nerve during operative dissection and decompression". If such a hypothesis is correct, neurolysis should be able to improve results. On the contrary, in our patients the outcome was not modified by the surgical trauma created by neurolysis; and this is not supporting Adams' hypothesis.

3. All surgical procedures performed to treat trigeminal neuralgia are related to a percentage of complications and side effects. Literature data describe a mortality ranging from $0.5 \%$ to $1.7 \%[11,13,19]$ regarding MVD procedures. The rate of CSF fistulas varies from $1.5 \%$ to $20 \%$ in absence of long-term sequelae [11]. The vast majority of long-term complications are related to cranial nerves deficits. Barker et al. [10] described permanent cranial nerves deficits in $2.9 \%$ of cases. Hypoesthesias in the fifth cranial nerve territories have been described in $0.09 \%-59 \%$ of cases $[10,18,20,23$,
29] and eighth cranial nerve deficits in $1.3 \%-23.8 \%$ of cases $[10,11,13,20,29,30]$. Seventh cranial nerve deficits have been described in $2 \%-3 \%$ of patients [11, 29]. The higher rates of cranial nerve deficits are reported in those conditions usually enhancing the surgical morbidity of MVD procedures, such as in the case of neurovascular conflicts difficult to correct (i.e. depending upon a megadolichobasilar artery) [20] or in case of reoperations performed to treat pain recurrence [21, 31-33]. Trigeminal dysesthesia occurs in 1.3\%-19\% of patients, while no cases of trigeminal anesthesia dolorosa have been described [10, 20, 21, 23, 29]. The risk of other major complications (intracranial hemorrhages, meningitis) ranges from $3 \%$ to $10 \%$ [11]. In our cases, notwithstanding a total incidence of major perioperative complications similar to literature data, we found an inferior rate of cranial nerve deficits at the long-term follow-up (trigeminal hypoesthesia, 6.4\%; eighth cranial nerve deficit, $1.2 \%$; seventh cranial nerve deficit, $0.6 \%$ ); no cases of dysesthesia or trigeminal anesthesia dolorosa were observed in our patients. Percutaneous procedures, although related to almost no surgical mortality, are plagued by a large number of functionally important and often untreatable complications. Trigeminal dysesthesias are reported in $6.7 \%-24.2 \%$ of cases after TCG procedures $[5,16,11$, $34]$ and in $6 \%-10.6 \%$ of cases after PMC [3, 8, 11, 16, 34]. $0.2 \%-9 \%$ of patients submitted to TCG and $0 \%-1.3 \%$ of patients who undergo PMC show areas of painful anesthesia $[11,16]$. Regarding PMC procedures, corneal areflexia appears as a less frequent long-term complication (PMC, 1.5\%-2.6\%; TCG, 15\%-23\%), while a higher rate of masticatory muscles deficit is observed (PMC, 1.3\%-66\%; TCG, 0\%-40\%); such deficits are often transitory and improve spontaneously within 6 months [11, 16, 34, 35]. Taha and Tew [11] proposed that more circumscribed lesions, focused on the areas affected by pain, permit to remarkably reduce trigeminal sensitive complications related to TCG procedures. These authors observed major areas of dysesthesia in $2 \%$ of patients, while areas of anesthesia dolorosa appeared in $0.2 \%$ of cases. In our experience, the incidence of major dysesthesias was $5 \%$ after PMC and $17.6 \%$ after TCG.

It is quite clear, looking at our results, that even though acute success rates could be considered acceptable for all the utilized procedures, long-term results are acceptable only for MVD. This analysis does not imply that percutaneous procedures are not of clinical utility. Many patients can be treated by percutaneous maneuvers, but will need many procedures and continuous care. Other cases will need different types of operations and every patient should be involved in the choice of his treatment 
after a clear and detailed analysis of the risks and results of each choice. We found the same outcome from MVD when performed as first or second treatment. Some of our patients, after this observation, decided to first undergo a percutaneous procedure and if pain recurred after a short time, they asked for MVD.

\section{References}

1. Loeser JD (1984) Tic doloureux and atypical face pain. In: Wall PD, Melzack R (eds) The textbook of pain. Churchill Livingstone, Edinburgh, pp 426-434

2. Jannetta PJ (1967) Arterial compression of the trigeminal nerve at the pons in patients with trigeminal neuralgia. $\mathbf{J}$ Neurosurg 26:159-162

3. Broggi G, Franzini A, Giorgi C, Servello D, Brock S (1993) Trigeminal neuralgia: new surgical strategies. Acta Neurochir Suppl (Wien) 58:171-173

4. Slettebo H, Hirschberg H, Lindegaard KF (1993) Long-term results after percutaneous retrogasserian glycerol rhizotomy in patients with trigeminal neuralgia. Acta Neurochir (Wien) 122(3-4):231-235

5. Zakrzewska JM, Thomas DG (1993) Patient's assessment of outcome after three surgical procedures for the management of trigeminal neuralgia. Acta Neurochir (Wien) 122(3-4):225-230

6. Kondziolka D, Lunsford LD, Bissonette DJ (1994) Long-term results after glycerol rhizotomy for multiple sclerosis-related trigeminal neuralgia. Can J Neurol Sci 21(2):137-140

7. Walchenbach R, Voormolen JH, Hermans J (1994) Microvascular decompression for trigeminal neuralgia: a critical reappraisal. Clin Neurol Neurosurg 96(4):290-295

8. Abdenebi B, Bouatta F, Chitti M, Bougatene B (1995) Percutaneous balloon compression of the gasserian ganglion in trigeminal neuralgia. Longterm results in 150 cases. Acta Neurochir (Wien) 136(1-2):72-74

9. Bergenheim AT, Hariz MI (1995) Influence of previous treatment on outcome after glycerol rhizotomy for trigeminal neuralgia. Neurosurgery 36(2):303-310
10. Barker FG 2nd, Jannetta PJ, Bissonette DJ, Larkins MV, Jho HD (1996) The long-term outcome of microvascular decompression for trigeminal neuralgia. N Engl J Med 334(17):1077-1083

11. Taha JM, Tew JM Jr (1996) Comparison of surgical treatments for trigeminal neuralgia: reevaluation of radiofrequency rhizotomy. Neurosurgery 38(5):865-871

12. Barker FG 2nd, Jannetta PJ, Bissonette DJ, Jho HD (1997) Trigeminal numbness and tic relief after microvascular decompression for typical trigeminal neuralgia. Neurosurgery 40(1):39-45

13. Kondo A (1997) Follow-up results of microvascular decompression in trigeminal neuralgia and hemifacial spasm. Neurosurgery 40(1):46-52

14. Sweet WH, Wepsic JG (1974) Controlled thermocoagulation of the trigeminal ganglion and rootlets for differential destruction of pain fibers. Part I: trigeminal neuralgia. J Neurosurg 40:143-156

15. Mullan S, Lichtor T (1983) Percutaneous microcompression of the trigeminal ganglion for trigeminal neuralgia. J Neurosurg 59:1007-1012

16. Apfelbaum RI (1994) Neurosurgical procedures on the trigeminal nerve and ganglion. In: Levy R, North R (eds) Neurosurgery of chronic pain. Williams and Wilkins, Baltimore, pp $1-46$

17. Oturai AB, Jensen K, Eriksen J, Madsen F (1996) Neurosurgery for trigeminal neuralgia: comparison of alcohol block, neurectomy and radiofrequency coagulation. Clin J Pain 12(4):311-315

18. Burchiel KJ, Clarke H, Haglund M, Loeser JD (1988) Long term efficacy of microvascular decompression in trigeminal neuralgia. J Neurosurg 69:35-38
19. Cho DY, Chang CGS, Wang YC, Wuang FH, Shen CC, Yang DY (1994) Repeat operations in failed microvascular decompression for trigeminal neuralgia. Neurosurgery 35(4):665-670

20. Linskey ME, Jho HD, Jannetta PJ (1994) Microvascular decompression for trigeminal neuralgia caused by vertebrobasilar compression. J Neurosurg 81(1):1-9

21. Rath SA, Klein HJ, Richter HP (1996) Findings and long-term results of subsequent operations after microvascular decompression for trigeminal neuralgia. Neurosurgery 39(5):933-940

22. Moller AR (1991) The cranial nerve vascular compression syndrome: a review of treatment. Acta Neurochir (Wien) 113:18-23

23. Steiger HJ (1991) Prognostic factors in the treatment of trigeminal neuralgia. Analysis of a differential therapeutic approach. Acta Neurochir (Wien) 113:11-17

24. Molina-Foncea A, Garcia-Navarrete E, Calvo JC, Sola RG (1988)

Microvascular decompression in the treatment of essential trigeminal neuralgia. Rev Neurol 27(155):65-70

25. Barba D, Alksne JF (1984) Success of microvascular decompression with and without prior surgical therapy for trigeminal neuralgia. J Neurosurg 60(1):104-107

26. Klun B (1992) Microvascular decompression and partial sensory rhizotomy in the treatment of trigeminal neuralgia: personal experience with 220 patients. Neurosurgery 30(1):49-52

27. Sun T, Saito S, Nakai O, Ando T (1994) Long-term results of microvascular decompression for trigeminal neuralgia with reference to probability of recurrence. Acta Neurochir (Wien) 126(2-4):144-148 
28. Adams CBT (1989) Microvascular compression: an alternative view and hypothesis. J Neurosurg 57:1-12

29. Bederson JB, Wilson CB (1989) Evaluation of microvascular decompression and partial sensory rhizotomy in 252 cases of trigeminal neuralgia. J Neurosurg 71:359-367

30. Moller AR, Moller MB (1989) Does intraoperative monitoring of auditory evoked potentials reduce incidence of hearing loss as a complication of microvascular decompression of cranial nerves? Neurosurgery

24(2):257-263
31. Liao JJ, Cheng WC, Chang CN, Yang JT, Wei KC, Hsu YK, Lin TK (1997) Reoperation for recurrent trigeminal neuralgia after microvascular decompression. Surg Neurol 47(6):562-570

32. Meglio M, Cioni B, Moles A, Visocchi M (1990) Microvascular decompression versus percutaneous procedures for typical trigeminal neuralgia: personal experience. Stereotact Funct Neurosurg 54:76-79
33. Mendoza N, Illingworth RD (1995) Trigeminal neuralgia treated by microvascular decompression: a long term follow-up study. Br J Neurosurg 9:13-19

34. Meglio M, Cioni B (1989)

Percutaneous procedures for trigeminal neuralgia: microcompression versus radiofrequency thermocoagulation. Personal experience. Pain 38:9-16

35. Meglio M, Cioni B, D’Annunzio V (1987) Percutaneous microcompression of the gasserian ganglion: personal experience. Acta Neurochir Suppl 39:142-143 